\title{
Applications of amorphous track structure models for correction of ionization quenching in organic scintillators exposed to ion beams
}

\author{
Christensen, Jeppe Brage; Andersen, Claus E.
}

Published in:

Radiation Measurements

Link to article, DOI:

10.1016/j.radmeas.2019.01.003

Publication date:

2019

Document Version

Peer reviewed version

Link back to DTU Orbit

Citation (APA):

Christensen, J. B., \& Andersen, C. E. (2019). Applications of amorphous track structure models for correction of ionization quenching in organic scintillators exposed to ion beams. Radiation Measurements, 124, 158-162. https://doi.org/10.1016/j.radmeas.2019.01.003

\section{General rights}

Copyright and moral rights for the publications made accessible in the public portal are retained by the authors and/or other copyright owners and it is a condition of accessing publications that users recognise and abide by the legal requirements associated with these rights.

- Users may download and print one copy of any publication from the public portal for the purpose of private study or research.

- You may not further distribute the material or use it for any profit-making activity or commercial gain

- You may freely distribute the URL identifying the publication in the public portal 


\title{
Applications of amorphous track structure models for correction of ionization quenching in organic scintillators exposed to ion beams
}

\author{
Jeppe Brage Christensen, Claus E. Andersen \\ Center for Nuclear Technologies, Technical University of Denmark, 4000 Roskilde, Denmark
}

\begin{abstract}
The scintillation response of organic plastic scintillators irradiated with heavy ions is investigated with the opensource code ExcitonQuenching. The software relies on amorphous track structure theory to account for the radial energy deposition by secondary electrons (EDSE) in ion tracks. The kinematic Blanc model is applied to evaluate the ionization quenching for a given ion by taking the decay time, light yield, and density of the scintillator into account. ExcitonQuenching predicts the scintillation response without a priori knowledge of any measured response curves in contrast to other EDSE models, such as the correction method due to Birks, which rely on free fitting parameters for each ion. ExcitonQuenching is validated against published measurements of the Pilot-U scintillator exposed to several ions. The agreement with experimental data is between $5 \%$ and $9 \%$ for ions with atomic number $z \leq 6$ but deviates significantly for heavier ions.
\end{abstract}

Keywords: Ionization quenching, organic plastic scintillators, ion beam dosimetry, amorphous track structure theory, particle therapy

\section{Introduction}

Water equivalent organic plastic scintillator detectors coupled to optical fibers for remote read out (Beddar et al., 1992a,b) enable measurements of absorbed dose with minimal perturbation and a high degree of resolution in time $(<1 \mathrm{~ms})$ and space (sub-mm). While both the temperature variations (Buranurak et al., 2013) of the scintillator and the radiation induced stem-effect in the fibre (Archambault et al., 2005) are well-understood and may be corrected for, the scintillators suffer in line with other solid state detectors from a response that is nonproportional to the local energy deposition. The reduced scintillator response occurring at high ionization densities, termed ionization quenching or nonproportionality, remains a challenge. Several kinematic models have been proposed to correct ionization quenching in inorganic scintillators (Michaelian and MenchacaRocha, 1994; Vasil'ev, 2008; Williams et al., 2010) but few have been suggested for organic materials.

Birks (1951) suggested a semi-empirical model to correct the scintillator signal for ionization quenching which later was extended by Chou (1952) for particles

Email address: jepb@dtu.dk (Jeppe Brage Christensen) with a high linear energy transfer (LET) with an additional free parameter. Nonetheless, neither of the models account for the local ionization density in ion tracks. The radial energy deposition by secondary electrons (EDSE) in the tracks of two ions with the same LET, but different atomic numbers, differ due to the ranges of the liberated secondaries. The differing ionization densities in the ion tracks thus cause the scintillation responses to differ as experimentally observed by Buenerd et al. (1976). Consequently, the Birks and Chou models require experimentally determined correction factors for each particle and each scintillator in question.

Michaelian and Menchaca-Rocha (1994) approached the ionization quenching issue with a model based on the EDSE and were as such capable of distinguishing between the quenching from different particles with the same LET. However, their model requires determination of several free quenching parameters by fitting the model to experimentally obtained data. The present work investigates the numerical framework ExcitonQuenching (Christensen and Andersen, 2018) for the case of plastic scintillators exposed to ions heavier than protons.

The software combines amorphous track structure theory and the decay time, light yield, and density of 
the scintillator to estimate the local ionization densities, upon which the scintillator response is evaluated through a kinematic model. ExcitonQuenching is in contrast to other EDSE models able to predict the quenched scintillator response without fitting parameters to specific response curves and can furthermore quantify the temporal structure of ionization quenching. The open-source software ExcitonQuenching is accessible for download ${ }^{1}$ to correct the ionization quenching in organic plastic scintillators exposed to ions.

\section{Methods}

\subsection{Ionization quenching}

The luminescence $\mathrm{d} L$ per unit length $\mathrm{d} x$ as a function of a particle's linear energy transfer is often written as

$$
\frac{\mathrm{d} L}{\mathrm{~d} x}=\frac{A \cdot \mathrm{d} E / \mathrm{d} x}{\mathrm{QCF}}, \quad \mathrm{QCF}=1+k B \cdot \mathrm{d} E / \mathrm{d} x,
$$

where $A$ denotes the light yield of the scintillator in units of photons emitted per deposited energy, and the quenching correction factor (QCF) is expressed as a linear function with slope $k B$ in the Birks model. The scintillator response $L$ for a particle with kinetic energy $E$ may consequently be computed from eq. (1) as

$$
\frac{\mathrm{d} L}{\mathrm{~d} E}=A \mathrm{QCF}^{-1} \Rightarrow L=\int_{0}^{E} A \mathrm{QCF}^{-1} \mathrm{~d} E
$$

for a thick scintillator. The QCF for a given ion and scintillator is a function of the kinetic energy and the EDSE which can be achieved through a track structure.

\subsection{Amorphous track structures}

Amorphous track structure theory models the EDSE as a continuous radial function and thus predicts the local ionization density based on the kinetic energy of the primary particle and the material composition of the medium. The present work focuses on the ChatterjeeSchaefer track structure model (Chatterjee and Schaefer, 1976), which consists of a dense core region of enormous ionization density and a penumbral region with the characteristic $r^{-2}$ radial decrease. The ChatterjeeSchaefer track structure model is defined in eq. (A.1) and illustrated in figure 1 for different ions with the same LET. As the ion core regions may exhibit huge excitation densities for slow, heavy ions, the ChatterjeeSchaefer results are compared to the Scholz-Kraft track structure model (Scholz and Kraft, 1996) to evaluate the influence of the applied track structure model. The Scholz-Kraft track structure model is outlined in eq. (A.2).

\footnotetext{
${ }^{1}$ https://github.com/jbrage/ExcitonQuenching
}

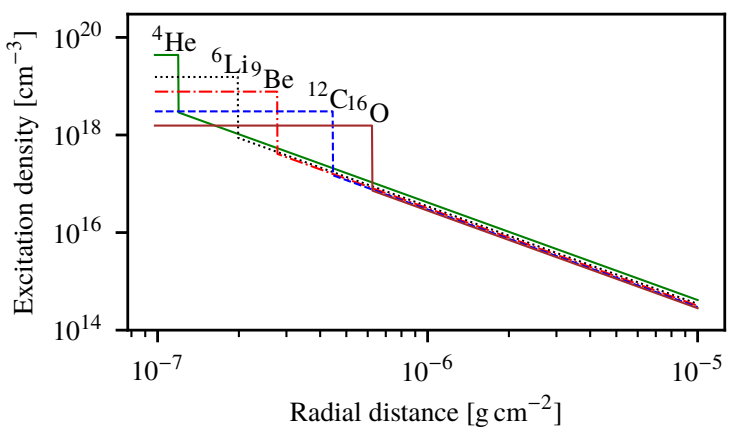

Figure 1: Close-up of the track structures of five ions with $\mathrm{d} E / \mathrm{d} x=$ $30 \mathrm{keV}_{\mu \mathrm{m}}^{-1}$ in the Pilot-U scintillator modelled as suggested by Chatterjee and Schaefer (1976).

\subsection{Kinematics of excited states}

An ion depositing an energy $\mathrm{d} E$ per unit length $\mathrm{d} x$ in a scintillator gives rise to a linear density of $N=\mathrm{d} E / \mathrm{d} x \cdot A$ of excited states (excitons). The exciton distribution immediately after an ion traversed the material slab of unit length $\mathrm{d} x$ is radially distributed according to the Chatterjee-Schaefer track structure.

The excitons of density $n$ in the ion track are in a kinematic model suggested by Blanc et al. $(1962,1964)$ allowed to diffuse, fluoresce, and quench according to

$$
\frac{\partial n}{\partial t}=D \nabla^{2} n-(p+k) n-\alpha n^{2},
$$

where the diffusion constant $D$ and bimolecular quenching parameter $\alpha$ are estimated from fits in Christensen and Andersen (2018) and listed in table 1. The unimolecular quenching parameter $k$ and the rate of fluorescence emission $p$ are related to the decay time $\tau$ of the scintillator via $\tau^{-1}=p+k$ (Birks, 1964) and weighted equally. Eq. (3) models the fluorescence emission from the first excited singlet state to the ground state (Blanc et al., 1962).

Table 1: The generic model parameters are calculated in Christensen and Andersen (2018) whereas the decay time $\tau$, light yield $A$ (in percent relative to anthracene), and density $\rho$ for the Pilot-U plastic scintillator are given by the manufacturer (Eljen Technology, USA).

\begin{tabular}{cccccc}
\hline \multicolumn{2}{c}{ Generic parameters } & & \multicolumn{3}{c}{ Scintillator parameters } \\
\cline { 1 - 2 } \cline { 5 - 6 } $\begin{array}{c}D \\
{\left[\mathrm{~cm}^{2} \mathrm{~s}^{-1}\right]}\end{array}$ & $\begin{array}{c}\alpha \\
{\left[\mathrm{cm}^{3} \mathrm{~s}^{-1}\right]}\end{array}$ & & $\begin{array}{c}\tau \\
{[\mathrm{ns}]}\end{array}$ & $\begin{array}{c}A \\
{[\%]}\end{array}$ & $\begin{array}{c}\rho \\
{\left[\mathrm{g} \mathrm{cm}^{-3}\right]}\end{array}$ \\
\cline { 1 - 2 } \cline { 5 - 7 } & $1.0 \times 10^{-9}$ & & 1.4 & 67 & 1.023 \\
\hline
\end{tabular}

\subsection{Quenching correction factors}

The kinematic eq. (3) governs the exciton interactions where the initial exciton distribution is given by 
the track structure model and scintillator light yield and material composition. The emitted light is calculated by solving eq. (3) numerically (see Christensen and Andersen (2018) for details) while integrating over the fluorescence term $p n$. Solving eq. (3) with $\alpha=0$ gives the light emission in the absence of ionization quenching while solving the same equation with $\alpha \neq 0$ gives the quenched scintillator response. The corresponding QCF can consequently be obtained as the ratio of the unquenched signal to the quenched signal.

The following section applies ExcitonQuenching to investigate the ionization quenching in a plastic scintillator (Pilot-U, Eljen Technology, USA) which was subject to irradiation with several ions heavier than protons by Buenerd et al. (1976). The results are obtained as predictions without fitting free parameters to experimentally determined scintillator responses for the Pilot$\mathrm{U}$ scintillator in contrast to the Birks model and most EDSE models.

\section{Results}

\subsection{Ionization quenching}

The QCF is calculated with ExcitonQuenching for ${ }^{4} \mathrm{He},{ }^{6} \mathrm{Li},{ }^{9} \mathrm{Be},{ }^{12} \mathrm{C}$, and ${ }^{16} \mathrm{O}$ ions for a wide range of $\mathrm{ki}-$ netic energies in the Pilot-U scintillator, where the LET as a function of energy for a given ion and material composition is calculated as suggested by Bird and Williams (1990). The QCFs as a function of LET is shown in figure 2 where the Birks model in eq. (1) has been fitted to each set of calculated QCFs.

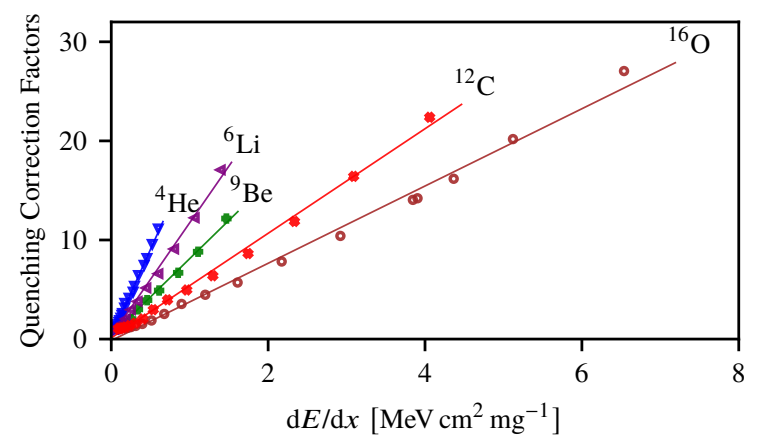

Figure 2: The quenching correction factor as a function of LET for five ions is calculated with ExcitonQuenching plotted with symbols. The Birks model is fitted to each set of data shown with solid lines.

The scintillation efficiency $\mathrm{d} L / \mathrm{d} E$ for the five ions are calculated according to eq. (2) as a function of the QCF which in turn depends on the kinetic energy of the particle. The results are shown in figure 3 as a function of kinetic energy.

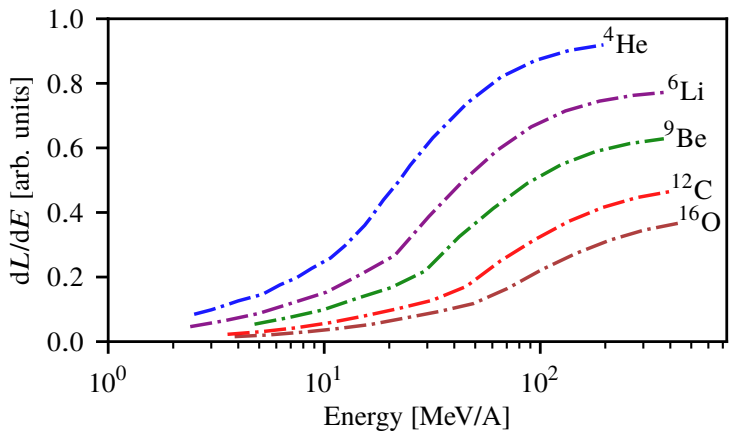

Figure 3: Theoretical light emission per energy as a function of kinetic energy in eq. (2) for the Pilot-U scintillator irradiated with different ions.

\subsubsection{Light emission for isotopes}

The scintillation efficiency for three isotopes of hydrogen, helium, and lithium are in figure 4 calculated as a function of velocity $\beta=v / c$ and velocity per atomic number $\beta / z$, respectively. The scintillation efficiency converges in all cases towards a quenching-free level for $\beta \rightarrow 1$, corresponding to $\mathrm{QCF} \rightarrow 1$ in eq. (2).

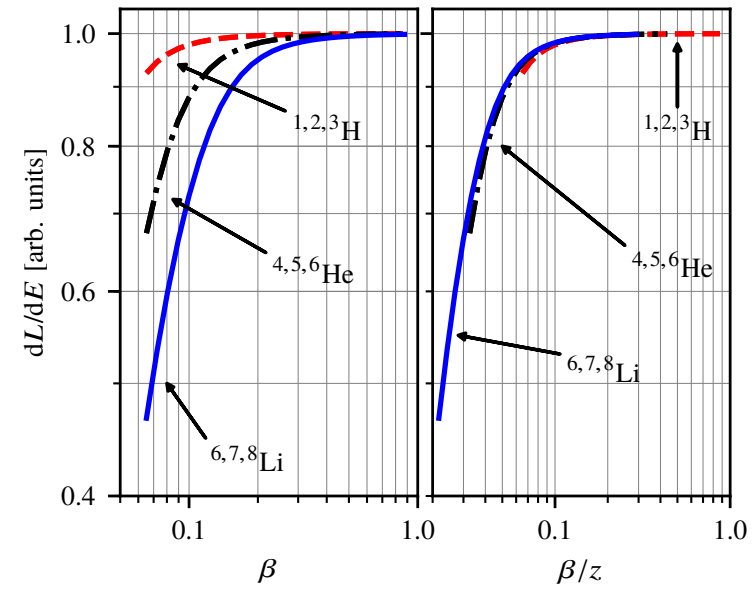

Figure 4: The scintillation efficiency $\mathrm{d} L / \mathrm{d} E$ for 3 isotopes of hydrogen, helium, and lithium as a function of (left) velocity and (right) velocity per atomic number $z$. All efficiencies coincide in the latter case.

\subsection{Light output}

The luminescence response from the plastic scintillator induced by a stopped ion of kinetic energy $E$ is calculated by integrating $\mathrm{d} L / \mathrm{d} E$ over the kinetic energy as given by eq. (2), where the QCF as a function of kinetic energy is interpolated for a continuous integration. The theoretical results are compared to the experimental results obtained by Buenerd et al. (1976) in figure 5, 
where the results are collectively scaled to the experimental data with a single value. The light output is calculated for all ions using the Chatterjee-Schaefer and Scholz-Kraft track structures where the isotopes ${ }^{7,8} \mathrm{Li}$ and ${ }^{9} \mathrm{Be}$ are shown for reference.

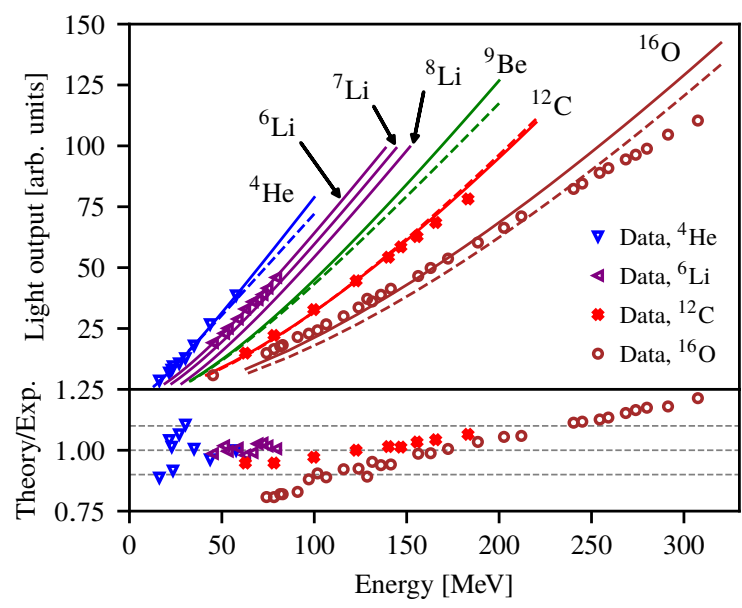

Figure 5: The light emission as a function of kinetic energy for ions stopping in the plastic scintillator. The calculated ExcitonQuenching results are shown with solid lines (ChatterjeeSchaefer track structure) and dashed lines (Scholz-Kraft track structure), whereas the experimental data points from Buenerd et al. (1976) with symbols as given by the legend. The luminescence is shown for three isotopes of lithium for comparison. The ratios between the ExcitonQuenching calculated responses (solid lines) to the experimentally measured scintillation responses are shown below where the dashed horizontal lines show $\pm 10 \%$ discrepancies.

\section{Discussion}

\subsection{Track structure theory}

The ion core densities for the five different ions in

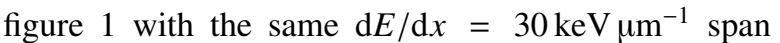
almost two orders of magnitude and illustrate the importance of the track structure models; the oxygen ion $(E \approx 190 \mathrm{MeV} / \mathrm{A})$ liberates secondaries with significantly larger kinetic energies than the $\alpha$ particle $(E \approx$ $5.5 \mathrm{MeV} / \mathrm{A})$. The core and penumbra radii in the oxygen ion track are consequently broader which in turn reduce the ionization density and quenching. The variation of the QCFs as a function of $\mathrm{d} E / \mathrm{d} x$ in figure 2 illustrates how the ionization quenching varies with the atomic number and $\mathrm{d} E / \mathrm{d} x$ as experimentally confirmed by Newman and Steigert (1960).

\subsection{Scintillation efficiency}

The scintillation efficiency in figure 3 as a function of energy shows how lighter ions more efficiently convert energy into luminescence for a given energy. The scintillation efficiency for each ion slowly converges as the kinetic energy increases in turn reducing the ionization density of core region and quenching, which also was observed experimentally (Murray and Meyer, 1961).

The luminescence responses for the isotopes of a given element coincide as a function of velocity in figure 4, which also was experimentally confirmed by Avdeichikov et al. (2002) for the case of BGO exposed to light ions. The scintillation efficiency as a function of velocity per atomic number is approximately the same for all ions as explained and measured by Sibczynski et al. (2018) for GAGG:Ce excited by alpha particles.

\subsection{Light emission}

The scintillation response curves in figure 5 , as ions are stopped in the plastic scintillator, illustrate the variation of luminescence efficiency with atomic number. The emitted light as a function of kinetic energy differs between isotopes of the same element, as illustrated in the figure with the 3 isotopes of lithium, which is in line with the experimentally measured responses reported by Avdeichikov et al. (2002) and figure 4.

The agreement between the ExcitonQuenching calculated and experimentally measured scintillation response curves for helium, lithium, and carbon ions confirms the application of track structure theory and the kinematic Blanc model to compute the ionization quenching for $z \leq 6$ ion beams. However, the deviation between the predicted and measured response for oxygen ions (as much as $\pm 25 \%$ ) in figure 5 exhibits a structure where the response is underestimated for low energies and overestimated for high energies. The magnitude and structure of the discrepancy between the two track structure models and the data are similar, implying that the deviation between theory and experimental data is related to the model itself and not the particular amorphous track structure model in question.

The kinematic Blanc model in eq. (3) governs the fluorescence emission from de-excitation of the first singlet state but does not account for triplet state interactions. Two triplet states may interact and give rise to one molecule in the ground state and one molecule in the singlet state and as such leading to fluorescence emission at a slower time scale than normally observed (Michaelian and Menchaca-Rocha, 1994; Jain et al., 2009). Thus, the probability for a bimolecular interaction between two triplet states and subsequent fluorescence emission increases with the local ionization density and occurs more frequently in carbon and oxygen ion tracks than in lighter ion tracks as helium and lithium. The possibility of extending the kinematic 
Blanc model to include triplet state interactions was suggested by Blanc et al. (1964) but is out of the scope of the present work.

Another explanation of the deviation may be found in the current equation itself, where higher-order density terms may be required to balance the high-LET ions. Such a conclusion is in line with Torrisi (2000) where the Birks model with an additional free parameter, i.e. the Chou model, was concluded to provide a better fit to the data for high-LET.

Conversely, the good agreement between data and ExcitonQuenching for $z \leq 6$ ions matches the results in Christensen and Andersen (2018), where the software accurately predicted the scintillation response for another plastic scintillator (BCF-60, Saint-Gobain, France) irradiated with protons.

\section{Conclusion}

The open-source software ExcitonQuenching predicts the quenched scintillation response in plastic scintillators exposed to ion beams based on amorphous track structure theory and a kinematic exciton model. The agreement between the software and experimental data for helium, lithium and carbon ions is, except for one outlier, better than $9 \%$, and as such in line with traditionally reported experimental uncertainties. The large discrepancy between the model and data for oxygen ions indicates that an additional model parameter, along with the inclusion of triplet state interactions, are required for high-LET ions.

Nonetheless, ExcitonQuenching accurately predicts the ionization quenching in the organic plastic scintillator exposed to ions with $z \leq 6$ based on the decay time, light yield, and density of the scintillator without any fit to the data. Such a predictive capability is in sharp contrast to most available EDSE models which contain free parameters requiring fits to each set of experimental data.

\section{Acknowledgement}

The work was supported by the Danish Cancer Society and the Danish Council for Independent Research (grant FTP, DFF 4184-00151). The authors wish to thank Dr. Fahed M. Alsanea, MD Anderson Cancer Center, for discussions pertaining to ionization quenching and EDSE models.

\section{Appendix A. Amorphous track structure models}

Chatterjee-Schaefer. The track structure model due to Chatterjee and Schaefer (1976) consists of a dense core with radius defined as

$$
r_{\text {min }}=\beta_{\text {ion }} \times 11.6 \mathrm{~nm}
$$

where $\beta_{\text {ion }}=v / c$ is the ratio of the speed $v$ to the speed of light $c$. The outer border of the penumbra has a radius of

$$
r_{\max }=0.768 E-1.925 \sqrt{E}+1.257 \quad[\mu \mathrm{m}]
$$

with the kinetic energy $E$ in units of $\mathrm{MeV} /$ nucleon. The radial exciton density is given as

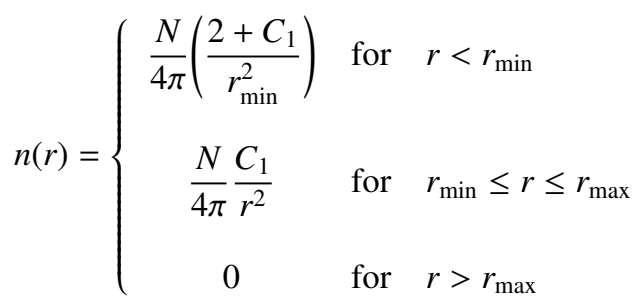

where

$$
C_{1}=\left(\ln \left(\sqrt{e} \frac{r_{\max }}{r_{\min }}\right)\right)^{-1}
$$

and $N=\mathrm{d} E / \mathrm{d} x \cdot A$.

Scholz-Kraft. With core and penumbral radii defined as

$$
r_{\max }=0.05 E^{1.7} \mu \mathrm{m}, \quad r_{\min }=0.01 \mu \mathrm{m},
$$

where $E[\mathrm{MeV} /$ nucleon $]$ is the kinetic energy of the projectile, the Scholz-Kraft track structure model governs a radial exciton density of

$$
n(r)=\left\{\begin{array}{cll}
\frac{C_{2}}{r_{\min }^{2}} & \text { for } \quad r<r_{\min } \\
\frac{C_{2}}{r^{2}} & \text { for } \quad r_{\min } \leq r \leq r_{\max } \\
0 & \text { for } \quad r>r_{\max }
\end{array}\right.
$$

for

$$
C_{2}=N\left(\pi\left[1+2 \ln \frac{r_{\max }}{r_{\min }}\right]\right)^{-1} .
$$




\section{References}

Archambault L, Beddar AS, Gingras L, Roy R, Beaulieu L. Measurement accuracy and Cerenkov removal for high performance, high spatial resolution scintillation dosimetry. Medical Physics 2005;33(1):128-35.

Avdeichikov V, Jakobsson B, Nikitin V, Nomokonov P, Wegner A Systematics in the light response of BGO, CsI (Tl) and GSO (Ce) scintillators to charged particles. Nuclear Instruments and Methods in Physics Research Section A: Accelerators, Spectrometers, Detectors and Associated Equipment 2002;484(1-3):251-8.

Beddar AS, Mackie TR, Attix FH. Water-equivalent plastic scintillation detectors for high-energy beam dosimetry: I. Physical characteristics and theoretical considerations. Physics in Medicine and Biology 1992a;37(10):1883-900.

Beddar AS, Mackie TR, Attix FH. Water-equivalent plastic scintillation detectors for high-energy beam dosimetry: II. Properties and measurements. Physics in Medicine and Biology 1992b;37(10):1901-13.

Bird RC, Williams JS. Ion beams for materials analysis. Elsevier, 1990.

Birks JB. Scintillation from organic crystals: Specific flourescence and relative response to different radiation. Proc Phys Soc A 1951;64(10):874-7.

Birks JB. The Theory and Practice of Scintillation Counting: International Series of Monographs in Electronics and Instrumentation. volume 27. Elsevier, 1964.

Blanc D, Cambou F, de Lafond YG, de Lafond YG. Kinetics of the fast component of scintillation in a pure organic medium. Application to anthracene. Comptes rendus de l'Académie des Sciences Paris 1962;18:3187-9.

Blanc D, Cambou F, Lafond YGD. Etude cinétique de la scintillation dans les cristaux organiques purs. Journal de Physique 1964;25:319.

Buenerd M, Hendrie D, Jahnke U, Mahoney J, Menchaca-Rocha A Olmer C, Scott D. Response of pilot u scintillator to heavy ions. Nuclear Instruments and Methods 1976;136(1):173-7.

Buranurak S, Andersen CE, Beierholm AR, Lindvold LR. Temperature variations as a source of uncertainty in medical fiber-coupled organic plastic scintillator dosimetry. Radiation Measurements 2013;56:307-11.

Chatterjee A, Schaefer HJ. Microdosimetric structure of heavy ion tracks in tissue. Radiation and Environmental Biophysics 1976;13(3):215-27.

Chou CN. The Nature of the Saturation Effect of Fluorescent Scintillators. Physical Review Letters 1952;5(87):904-5.

Christensen JB, Andersen CE. Relating ionization quenching in organic plastic scintillators to basic material properties by modelling excitation density transport and amorphous track structure during proton irradiation. Physics in Medicine and Biology 2018;63:195010.

Jain A, Blum C, Subramaniam V. Chapter 4 - fluorescence lifetime spectroscopy and imaging of visible fluorescent proteins. In: Verdonck P, editor. Advances in Biomedical Engineering. Amsterdam: Elsevier; 2009. p. 147 -76.

Michaelian A, Menchaca-Rocha A. Ion-Induced Luminescence Based on Energy Deposition. Physical Review B 1994;49(22):550-62.

Murray R, Meyer A. Scintillation response of activated inorganic crystals to various charged particles. Physical Review 1961;122(3):815.

Newman E, Steigert FE. Response of $\mathrm{NaI}(\mathrm{Tl})$ to Energetic Heavy Ions. Physical Review 1960;118(6):1575-8.

Scholz M, Kraft G. Track structure and the calculation of biologica effects of heavy charged particles. Advances in space research: the official journal of the Committee on Space Research 1996;18(12):5-14.

Sibczynski P, Czarnacki W, Mianowska Z, Mianowski S, Moszynski M, Sworobowicz T, Swiderski L, Bezbakh AA, Fomichev AS, Krupko SA, Sabelnikov AV, Kamada K, Shoji Y, Yoshikawa A. Non-proportionality of GAGG:Ce scintillators down to $50 \mathrm{eV}$ electron equivalent by application of alpha particle excitation. $\mathrm{Nu}-$ clear Instruments and Methods in Physics Research Section A: Accelerators, Spectrometers, Detectors and Associated Equipment 2018;898:24-9.

Torrisi L. Plastic scintillator investigations for relative dosimetry in proton-therapy. Nuclear Instruments and Methods in Physics Research, Section B: Beam Interactions with Materials and Atoms 2000;170(3):523-30

Vasil'ev AN. From luminescence non-linearity to scintillation non-proportionality. IEEE Transactions on Nuclear Science 2008;55(3):1054-61.

Williams R, Li Q, Grim JQ, Ucer K, Bizarri G, Moses W. Role of carrier diffusion and picosecond exciton kinetics in nonproportionality of scintillator light yield. In: Hard X-Ray, Gamma-Ray, and Neutron Detector Physics XII. International Society for Optics and Photonics; volume 7805; 2010. p. 78050K 